\title{
Lumen
}

Selected Proceedings from the Canadian Society for Eighteenth-Century Studies

\section{La République des Lettres ou l'empire de la rhétorique : une question de préface}

\section{Elizabeth Zawisza}

Volume 12, 1993

URI : https://id.erudit.org/iderudit/1012584ar

DOI : https://doi.org/10.7202/1012584ar

Aller au sommaire du numéro

Éditeur(s)

Canadian Society for Eighteenth-Century Studies / Société canadienne d'étude du dix-huitième siècle

ISSN

1209-3696 (imprimé)

1927-8284 (numérique)

Découvrir la revue

Citer cet article

Zawisza, E. (1993). La République des Lettres ou l'empire de la rhétorique : une question de préface. Lumen, 12, 123-130. https://doi.org/10.7202/1012584ar

Copyright (c) Canadian Society for Eighteenth-Century Studies / Sociéte canadienne d'étude du dix-huitième siècle, 1993
Ce document est protégé par la loi sur le droit d'auteur. L'utilisation des services d'Érudit (y compris la reproduction) est assujettie à sa politique d'utilisation que vous pouvez consulter en ligne.

https://apropos.erudit.org/fr/usagers/politique-dutilisation/ 


\title{
11. La République des Lettres ou l'empire de la rhétorique: une question de préface.
}

\begin{abstract}
/.../Le goût du temps n'est plus celui de l'antiquité..., il est vrai que pour mériter autrefois à bon titre la qualité d'Auteur, il fallait un génie vaste, un jugement solide, une érudition profonde; mais aujourd'hui, sans tout cela, on se fait naturaliser dans la République des Lettres.-Gaillard de la Bataille, Préface de Jeannette seconde ou la nouvelle paysanne parvenue (1744)
\end{abstract}

Faut-il insister sur le bouleversement que subit l'espace littéraire à l'époque des Lumières? A l'âge classique déjà, la vieille République des Lettres latines, savante et érudite, se voit confrontée à de nouvelles Lettres, modernes et mondaines, et se transformera lentement mais non sans difficulté, en 'littérature,' au sens actuel du terme. ${ }^{1}$ C'est au XVIIIe siècle que ce nouveau concept de littérature, qui avait longtemps désigné la culture générale ('Chapelain avait une littérature immense,' dit Voltaire), s'enrichit soudain de sens autrement riches. Conçu comme ensemble de la production littéraire d'un pays ou d'une époque, analysé comme catégorie abstraite et intellectuelle, ${ }^{2}$ le mot littérature se charge de connotations socio-culturelles et politiques. Il désigne désormais un milieu littéraire spécialisé, une nouvelle République des Lettres, ${ }^{3}$ beaucoup plus démocratique que ne l'avait été l'ancienne 'res literaria.' Ceux qui la peuplent, hommes de lettres savants et érudits, aiment aussi à se proclamer 'membres utiles' (Montesquieu) ou 'concitoyens d'une immense république d'esprits cultivés' (Voltaire), d'une république dont le mot d'ordre de 'Liberté, vérité et pauvreté' (d'Alembert) annonce déjà la fameuse devise républicaine.

Mais une vision de l'activité littéraire ainsi envisagée n'est-elle pas la conséquence directe $d^{\prime}$ une théorie de la connaissance qui se proposerait, sans modestie, d'aborder l'univers dans sa totalité? Ce sentiment de puissance cognitive, Montesquieu le personnifie admirablement, lui qui, durant ses voyages, va 'toujours sur le plus haut clocher ou la plus haute tour, pour voir le tout ensemble. ${ }^{4}$ Et on sait à quel point une pareille prétention a conduit les écrivains à mettre en question la notion com- 
mune de l'écriture. Pour saisir l'ensemble des phénomènes reçus des sens, pour aborder le monde discontinu et un à la fois, le créateur se doit ici d'échapper aux limites étroites d'un genre ou d'une technique pour devenir, selon la formule d'A. Comte, un 'spécialiste des généralités.'

Ainsi le concept globalisant de République des Lettres, revu et corrigé par les Lumières dans un nouveau contexte culturel et idéologique, impose aux écrivains une réflexion sur les stratégies discursives les plus efficaces. Entre 'res literaria,' 'littérature' et 'genre romanesque,' entre 'homme de lettres,' 'auteur' et 'romancier,' la révolution esthétique, qui sépare ces notions, ne saurait évidemment prendre corps que dans des formes linguistiques concrètes. Vus dans cette perspective, le roman des Lumières et sa préface, notamment, font sentir les tensions qui mettent aux prises les techniques romanesques modernes, indices de nouvelles préoccupations littéraires, et la rhétorique, technique traditionnelle, dont témoigne toujours le discours théorique des introductions. Il est à espérer que l'analyse des procédés préfaciels, proposée dans ces pages, élucidera la stratégie de prise de parole la plus fréquente au XVIIIe siècle. Pour investir l'idée égalitaire de République des Lettres et imposer le concept moderne de littérature, le romancier fait d'abord une brèche dans l'empire de la rhétorique' (Ch. Perelman), en combattant l'ennemi classique à l'aide de ses propres armes.

Ainsi les Lumières ont à coeur de réhabiliter la préface, de lui accorder une place plus importante que celle qui demeure au 'seuil' de l'oeuvre. ${ }^{5}$ Le répétitif, la louange et le banal préfaciels, bref, tout ce qui dérange dans leur poétique comme trop 'personnel' et peu 'instructif,' renvoie en fait aux figures/stratégies de l'ancien exorde, première partie de chaque discours construit selon l'ordre rhétorique. Est-il dès lors étonnant que, parmi les lieux propres au démonstratif, au délibératif et au judiciaire, ce soit le judiciaire qui fournisse au préfacier le gros de ses procédés? Dans le contexte esthétique 'officiel' des Lumières, le romancier ne peut aspirer ni à un 'tête-à-tête' avec le lecteur ni à un 'tête-à-texte' avec son livre. Le roman se posant comme genre moralement et esthétiquement controversé, ${ }^{6}$ l'auteur dans son rôle de préfacier doit d'abord, pour argumenter et séduire en faveur de sa vision du roman, réfuter et disqualifier la thèse adverse, contre-discours antiromanesque classique. La préface des Lumières vire ainsi à la 'mise en scène' pour autrui, en manipulant 'un tiers contre la personne attaquée. ${ }^{8}$ Comme s'il était sur scène ou encore devant un tribunal, le préfacier donne à l'éternel conflit, qui semble opposer romancier, adversaire et public/juge, l'allure de procès ou de spectacle. Comme stratégie de défense, la préface exhibe toutes les particularités du discours argumentatif, doxologique, agonique et, ce qui est plus propre au XVIIIle siècle, polémique. 
Comme tout discours argumentatif, la préface a pour fonction première d'émouvoir et de persuader, et non de convaincre, comme seul le discours démonstratif saurait le faire. Elle ne cache d'ailleurs pas ces objectifs, au reste si mal jugés par le lecteur. L'introduction n'est-elle pas, tout comme l'exorde, le lieu d'énonciation où l'auteur, 'après s'être assuré un auditoire bienveillant ... s'emploie à lui dévoiler le sujet et à $l^{\prime} y$ intéresser? ${ }^{10}$ On comprend mieux dès lors pourquoi la préface ne peut établir aucune conclusion rigoureuse, aucun 'savoir objectif' sur le roman; son auteur y défend tout au plus une thèse probable, 'c'est-à-dire susceptible d'être acceptée comme vraisemblable, comme ayant les plus grandes chances d'être en accord avec la vérité' (Vignaux 7). Proposer une nouvelle vision du genre romanesque, pour laquelle on veut obtenir l'adhésion du public, telle semble être la mission fondamentale de la préface; son contenu n'est qu'une suite des stratégies argumentatives devant mener à bien un projet difficile. 'C'est encore les Ouvrages de cette nature, observe un préfacier, qui servent à polir nos moeurs et notre esprit. Mais j'entends déjà les gens austères se récrier: Quoi! désormais il nous faudra donc étudier la morale dans des Livres tissus d'avantures extraordinaires. Et pourquoi non, Messieurs? si ces Livres sont composés avec goût et avec jugement, les jeunes personnes en tireront tout autant d'avantages que de la lecture de ces Auteurs célebres.... ${ }^{11}$ Tout est dans cette introduction, typique, des Lumières: contre-discours résumé, argumentation le réfutant, et le verdict en suspension, toujours attendu de la part du lecteur. La préface dialectique, réponse à une attaque rapportée textuellement, ou inscrite en filigrane, s'érige ainsi en discours doxologique: elle 'reçoit en partie passivement l'opinion courante' (Angenot 33), la doxa classique farouchement antiromanesque.

Or, face à cette doxa, le préfacier ne peut se permettre de rester passif, sa cause n'étant pas évidente en soi. Parce qu'il n'est pas encore assez fort, il lui est impossible de contourner les arguments adverses dans un essai-diagnostic ou un essai-méditation; il opte alors pour un discours agonique, à double fonction: 'démonstration de la thèse et réfutation/disqualification d'une thèse adverse' (Angenot 34). Et comme les manuels de rhétorique lui offrent tout un éventail de stratégies, il choisit dans leur arsenal les plus efficaces, celles du discours polémique, au sens strict proposé par M. Angenot. Il reconnaît en effet que le discours adverse est 'justiciable de prémisses communes à partir desquelles il peut être réfuté' (Angenot 35); ce terrain commun, c'est la rhétorique et la poétique classique. Stratège adroit, l'écrivain des Lumières reprend les concepts et les procédés mêmes du discours ennemi pour les contester d'autant plus fortement et les transgresser.

L'accusation étant déjà formulée plusieurs fois (dans des traités, des arts poétiques, des journaux littéraires), chaque préface la réfute en 
quelque sorte 'par anticipation. ${ }^{12}$ Pour y parvenir, elle essaie de fournir $\mathrm{d}$ 'abord des preuves 'extrinsèques' (Crevier), 'sans artifice' (R. Bary): 'documents' et 'témoignages' objectifs en faveur du roman (Kibedi Varga 36). Dans des textes longs et élaborés, imitant les manifestes apparemment non polémiques, Boyer d'Argens, Mouhy, Béliard, Bricaire de la Dixmerie ${ }^{13}$ présentent, preuves à l'appui, l'histoire du genre romanesque, sa carrière dans d'autres pays, des voix de la critique pro-romanesque. Or, comme ces données objectives ne sont pas encore très nombreuses, aux preuves 'extrinsèques' s'ajoutent vite, dans les préfaces, les preuves 'intrinsèques' ('artificielles'), où 'le matériel est transformé en force persuasive par une opération logique, ${ }^{14}$ ainsi que par les moyens insidieux, 'affectifs' (la captatio benevolentiae).

Opération logique, la préface peut se baser soit sur l'induction (l'exemple) soit sur la déduction (l'enthymème); les deux prennent une forme linguistique concrète grâce aux lieux (communs et propres), catégories formelles, qui fournissent le contenu aux arguments. Il semblerait même que la préface, à s'apparenter au panégyrique, tend à recourir à la louange, lieu propre au genre démonstratif. Mais, quand un préfacier oppose aux 'vilains Livres,' remplis 'd'équivoques,' 'd'une indécence affreuse,' son roman 'raisonnable,' 'ouvrage de sentiment' 'écrit avec beaucoup de naturel et de dignité, ${ }^{15}$ il atteste la démarche judiciaire typique de 'l'exemple,' qui érige le roman préfacé en 'précédent.' Faisant fond sur la louange, le romancier voudrait qu'on prenne son texte pour 'un exemple fondant une [nouvelle!] règle' (Perelman 472) sur l'utilité esthétique et morale du genre romanesque. S'il échoue dans sa tentative, c'est parce que sa tactique, rhétoriquement parlant, laisse à désirer. Selon les rhéteurs, l'auteur peut tirer de quatre sources les arguments en faveur de son oeuvre: 'de sa personne,' 'de la personne des clients,' 'de la personne de l'adversaire' ou encore du sujet lui-même. ${ }^{16}$ A cette époque, aucune de ces sources ne fournissait de matière solide pour défendre le roman, car aucune n'était opératoire comme argument 'par autorité'! Le romancier et le liseur de romans étaient alors jugés de 'têtes faibles' et d"esprits frivoles' (Voltaire), l'adversaire (critique, censeur, moraliste) attendait des 'preuves' et des 'témoignages,' et le roman lui-même constituait, comme type de discours, une 'cause ambiguë.'

L'exemple offrant une persuasion trop 'douce,' le préfacier se tourne vers les lieux les plus forts du judiciaire: l'enthymème, forme rhétorique du syllogisme, et les états de la question. Sa préface imite une démarche enthymématique pour répondre plus efficacement au leitmotiv de la critique classique: 'On s'y gâte le goût, on y prend de fausses idées de la vertu. ${ }^{, 17}$ Et ce lieu commun n'est-il pas la 'conclusion' d'un syllogisme dont la 'majeure' et la 'mineure' restent souvent sous-entendues? Le raisonnement complet s'impose: la majeure - les ouvrages de fiction sont 
esthétiquement et moralement blâmables; ${ }^{18}$ la mineure - or, le roman est une fiction; la conclusion - donc, le roman est esthétiquement et moralement blâmable. Le contenu conceptuel des préfaces des Lumières, mais aussi leurs tropes et figures, n'ont d'autre finalité que de réfuter au moins une de trois parties du syllogisme. Démarche qui se justifie $\mathrm{d}$ 'autant plus naturellement que les prémisses de l'enthymème rhétorique n'ont pas la force du 'certain scientifique' (R. Barthes), mais se basent entièrement sur le vraisemblable, sur ce que l'opinion commune accepte (Angenot 168 et passim). Ainsi, même si le public des Lumières reconnaît que la morale devrait être enseignée à partir d'exemples positifs, ${ }^{19}$ rien n'empêche le romancier d'opposer à la maxime communément admise une autre maxime morale. Sade ne se propose en effet rien d'autre lorsqu'il fait valoir le côté hautement instructif du tableau libertin.

$C^{\prime}$ est toujours la topique rhétorique qui aide le préfacier à combattre le syllogisme antiromanesque. Il peut attaquer la 'majeure,' en prouvant, par exemple, qu'elle pèche contre le lieu ontique de la non-contradiction. Car, si les ouvrages de fiction - et la comédie, la tragédie et l'épopée en font partie! - sont condamnables, pourquoi les Anciens, à l'autorité incontestable, les ont-ils pratiqués? La contre-attaque du romancier se réfère, elle aussi, aux lieux ontiques: lieu des semblables et lieu de l'acte à la personne. Un autre argument populaire est, par contre, issu du lieu déontique de justice: l'Histoire a beau présenter 'les inconvenances et les immoralités choquantes, ${ }^{20}$ on ne l'en valorise pas moins. Ou encore ce raisonnement préfaciel qui accule l'adversaire à l'absurdité: le postulat classique d'être moral et réaliste à la fois se révèle tout bonnement un dilemme insurmontable. ${ }^{21}$

Plusieurs préfaces préfèrent s'attaquer à la 'mineure' qui voit dans le roman la fiction. C'est ici que les trois formes de la question s'avèrent $d^{\prime}$ 'une extrême utilité, et leur apparition successive marque une évolution notable dans la 'théorie du roman.' L'auteur, qui n'ose pas encore se présenter comme producteur de fictions, offre au lecteur une 'Histoire véritable,' des 'Mémoires secrets' ou des 'Lettres' originales; il est 'l'accusé [qui] nie le fait: voilà l'état conjectural' (Kibedi Varga 56). Aussi le romancier de la deuxième moitié du XVIIIe siècle, qui se sent plus fort, opte-t-il pour l'état de qualité,' tel le vieil Horace, qui 'ne nie point que son fils ait tué sa fille, mais [qui] prétend que sa fille étant coupable, celui qui l'a tuée a fait une action de justice. ${ }^{22}$ Le préfacier fait remarquer en effet que si l'ouvrage de fiction est immoral et stylistiquement imparfait, c'est parce qu'il constitue une imitation parfaite de la réalité: les vices des hommes sont à l'origine du caractère 'vicieux' du genre romanesque! A tout dire cependant, la défense la plus courageuse, et qui n'est pas sans risques pour l'auteur, est l'état de la définition' où, tout en avouant le 'crime' qu'il a commis en écrivant un roman, il nie son méfait. 'J'ose dire 
que souvent l'histoire est un mauvais roman; et que le roman ... est une bonne histoire,' proclame Diderot, ${ }^{23}$ le plus audacieux des romanciers des Lumières. Et il suggère même qu'on change le nom du genre contesté!

$D^{\prime}$ autres stratégies préfacielles connues se révèlent comme des attaques directes de la 'conclusion' ('le roman est esthétiquement et moralement blâmable'). C'est ici qu'apparaissent tous les arguments qui, réfutant la lecture classique, sont du genre: argument du 'tableau de la vie humaine,' du 'traité de morale,' de la 'justice immanente,' des 'pièges dénoncés' (May), et toute réflexion sur la supériorité du style romanesque 'naturel' sur le style 'élevé.' En fait, dans son combat contre le syllogisme adverse, le romancier met en avant avec force une notion de la littérature nouvelle et qui annonce le romantisme et le réalisme. Toutefois, comme sa thèse n'émerge que de la confrontation avec une autre thèse, au demeurant elle aussi recevable, la préface s'érige en genre amphidoxon, dubium, où, nécessairement, 'il faut entraîner le juge à vous suivre comme guide' pour le rendre 'réceptif, malléable' (Barthes 215). Dans ce contexte, la captatio benevolentiae tant contestée de la préface, loin d'être une hyperbole gratuite, comme le veut J.L. Borges, ${ }^{24}$ se transforme dans la polémique en argument auxiliaire. Le 'Mon cher lecteur' d'un Villaret doit être perçu comme une technique 'favorisant la communion de l'orateur avec l'auditoire' (Perelman 431), et l'invective de Duclos dans la préface d'Acajou et Zirphile ${ }^{25}$ relève de la rhétorique 'de l'injure.'

Ainsi, dans le cas du roman, la survivance de la rhétorique au XVIIIe siècle signale paradoxalement une réalité esthétique nouvelle. En attendant son 'sacre, ${ }^{26}$ l'écrivain emprunte des outils discursifs à ses ennemis, stratégie qui témoigne de changements cruciaux au sein de la République des Lettres. Le romancier, face à la doxa et à ses formes d'énonciation, se voit obligé de construire son prestige sur une image aussi totalisante que celle qu' offrait la doctrine classique. Il propage une vision optimisted'une nouvelle République des Lettres peuplée d'écrivains et de genres égaux, qu'ils soient nobles ou bourgeois, ${ }^{27} l^{\prime}$ espace où le genre romanesque veut se tailler la partdulion. Dans sa préface, le romancier marginalisés'efforce de montrer qu'un 'terrain d'entente' existe, que l'échange d'égal à égal est possible du simple fait qu'il manie les mêmes armes que les adversaires. Au fond, si 'le roman modernenaîtauXVIIIe siècle' (H.Coulet), c'est parce que son créateur, l'ancien paria de la République des Lettres érudites, prouve bien que lui aussi peut s'emparer du discours doxologique pour faire du genre contesté un membre à part entière de la littérature. 


\section{Notes}

1 Voir M. Fumaroli, L'Age de l'éloquence: Rhétorique et 'res literaria' de la Renaissance au seuil de l'époque classique (Genève: Droz, 1980); A. Barnes, Jean Le Clerc (1657-1736) et la République des Lettres (Paris: Droz, 1938).

2 Comme dans Les siècles de la littérature française de Sabatier de Castres et dans les Éléments de littérature de Marmontel.

3 Pour l'évolution du terme 'littérature,' voir E. Escarpit, 'Histoire de l'histoire de la littérature,' Histoire des littératures, sous la réd. de R. Queneau, 3 vol. (Paris: Gallimard, 1978), 1: 1751 et passim.

4 Journal de voyage, cité par J. Starobinski, Montesquieu par lui-même (Paris: Seuil, 1963) 37.

5 Voir G. Genette, Seuils (Paris: Seuil, 1987).

6 Pour les grandes lignes de cette controverse, voir G. May, Le dilemme du roman au XVIIIe siècle (New Haven: Yale UP; Paris: PUF, 1963).

7 Cf. G. Vignaux, L'Argumentation: Essai d'une logique discursive (Genève, Paris: Droz, 1976) 72.

8 A. Greive, 'Comment fonctionne la polémique?' Le Discours polémique: Aspects théoriques et interprétations, sous la réd. de G. Roellenbleck (Tübingen, Paris: Gunter Narr Verlag, Ed. Jean-Michel Place, 1985) 19.

9 Je mets à profit l'appareil terminologique de M. Angenot, La parole pamphlétaire: Typologie des discours modernes (Paris: Payot, 1982).

10 A. Kibedi Varga, Rhétorique et littérature: Études de structures classiques (Paris: Didier, 1970) 72.

11 J.-B. Jourdan, 'Préface' au Guerrier philosophe (1744), cité par H. Coulet, Le Roman jusqu'à la Révolution, 2 vol. (Paris: A. Colin, 1968) 2: 144.

12 Cf. Ch. Perelman, L. Olbrechts-Tyteca, La nouvelle rhétorique: Traité de l'argumentation, 2 vol. (Paris: PUF, 1958) 2: 662.

13 Dans, respectivement, 'Discours sur les Nouvelles' (dans Lectures amusantes, ou les Délassements de l'esprit, 1739), 'Préface ou Essais pour servir de réponse à un ouvrage intitulé Entretiens sur les Romans, par M. l'abbé J.' (Le Financier, 1755), 'Préface ou discours pour servir à la défense des Romans (Zelaskim, 1765) et 'Discours sur l'origine, les progrès et le genre des Romans' (Toni et Clairette, 1793).

14 R. Barthes, 'L'ancienne rhétorique: Aide-mémoire,' Communications 16 (1970): 200.

15 Comme dans la préface à l'Histoire d'une femme de qualité (ouvrage anonyme, La Haye, 1749) III-VI.

16 J.-P. Papon, L'Art du poète et de l'orateur: Nouvelle rhétorique à l'usage des collèges (1768; Genève: Slatkine reprints, 1970), chap. 'Des sources de l'exorde.'

17 Bruzen de la Martinière cité par May 8.

18 'Un faiseur de romans et un poète de théâtre est un empoisonneur public...,' clame le janséniste Nicole.

19 Les fameuses 'vérités douces' de Mme Riccoboni!

20 Michel de Pure, La Prétieuse (Première Partie), cité par May 140.

21 C'est à ce dilemme que G. May a consacré son ouvrage déjà cité.

22 L'exemple du rhétoricien Soarez, cité par Kibedi Varga 56. 
23 Dans 'Éloge de Richardson,' cité par May 150.

24 Dans Livre de préfaces (Paris: Gallimard, 1980) 10.

25 'Vous parlez sans penser, vous agissez sans dessein, et vous croyez juger parce que vous prononcez. Je vous respecte beaucoup, je vous estime très peu: voilà mes sentiments à votre égard...,' dit-il dans son 'Epître au public' (Oeuvres de Duclos, 3 vol. [Paris: A. Belin, 1821] 1: 392).

26 Cf. P. Bénichou, Le sacre de l'écrivain 1750-1830 (Paris: José Corti, 1973).

27 F. Furet cité par E. Walter, 'L'intelligentsia des lumières,' Dix-huitième siècle 5 (1973): 179. 